\title{
Performance of surrogate models in reliability-based design optimization
}

\author{
M. Cid Montoya, J. Díaz \& S. Hernández \\ Structural Mechanics Group, School of Civil Engineering, \\ University of La Coruña, Spain
}

\begin{abstract}
Structural designs are progressively more conditioned by uncertainty in a wide range of fields, and new designs have to meet the requirements of safety and efficiency. Probabilistic optimization is a powerful tool able to improve and optimize initial designs into others which are more efficient. In spite of the continuous growth of computational power, this kind of optimization usually turns out to be unaffordable, due to the computational cost of the methods involved. However, parallel computing and surrogate models can overcome this drawback by reducing drastically the total computational cost of the process. In this paper, a survey of the performance of several surrogate models combined with reliabilitybased design optimization is presented. Two examples are selected to show the behavior of the methods when applied to different models.
\end{abstract}

Keywords: uncertainty quantification, reliability based design optimization, surrogate models.

\section{Introduction}

Reliability-based design optimization (RBDO) is a design technique that is applied typically in aerospace and automotive engineering. An intensive review can be found in [1-3]. This kind of optimization tries to minimize an objective function, which in structural optimization is usually related with the cost of the structure, subjected to probabilistic constraints. These probabilistic constraints involve solving a reliability analysis of the structure. Consequently, two types of variables can be found in the process. Design variables, which are modified in the optimization cycle, and random variables, which are the source of uncertainty. 
Besides, design variables can also be affected by uncertainty. These variables are defined as random design variables.

The formulation of a RBDO problem can be set out in different ways. Double loop or bi-level approaches consider the reliability within the optimization loop. This is the case of the reliability index approach (RIA) or the performance measure approach (PMA). In RIA, the outer loop considers non-deterministic constraints which are based on reliability indexes, while in the inner loop reliability indexes are obtained from defined limit state function. On the other hand, nondeterministic constraints of the PMA outer loop are based on limit state functions, whereas in the inner loop the limit state functions are obtained from defined reliability indexes. Mono-level approaches replace probabilistic constraints with approximate deterministic values, converting the double loop into a single loop. Decoupled approach solves the RBDO problem as a sequence of deterministic optimization procedures, like sequential optimization and reliability assessment (SORA) method. In this work, a double loop framework with PMA has been selected as methodology.

However, the application of these methods to time-consuming models sometimes leads to unaffordable computational times. In order to solve this problem, surrogate models emerge as a suitable alternative. There is a wide-range of surrogate methods, and some interesting information can be found in [4,5]. In this work, several surrogates models are used with RBDO in order to show its accuracy and computational improvements. A brief description of the surrogate algorithms employed in this work is presented next.

\section{Surrogate models}

\subsection{Polynomial}

Polynomial surrogate models are defined employing multivariate polynomial according to the general expression

$$
\hat{f}_{P}(\boldsymbol{x})=c_{0}+\sum_{k=1}^{p}\left(\sum_{i_{1}=1}^{n} \cdots \sum_{i_{k} \geqslant i_{k-1}}^{n} c_{i_{1} \cdots i_{k}} \prod_{j=1}^{k} x_{i_{j}}\right),
$$

where $\hat{f}_{p}$ is the response of the surrogate model, $p$ is the order of the polynomial, $n$ is the number of variables, $x_{i_{j}}$ are the components of the $n$-dimensional vector of variables and the terms $c_{0}$ and $c_{i_{1} \cdots i_{k}}$ are the polynomial coefficients.

\subsection{Kriging}

Kriging emulator $\hat{f}_{K}[6,7]$ can be expressed as

$$
\hat{f}_{K}(\boldsymbol{x})=\boldsymbol{\kappa}(\boldsymbol{x})^{T} \boldsymbol{\rho}+\varepsilon(\boldsymbol{x}),
$$


where $\boldsymbol{\kappa}(\boldsymbol{x})^{T} \boldsymbol{\rho}$ is a trend function, which usually is a low order polynomial, and $\varepsilon(\boldsymbol{x})$ is a stationary Gaussian process error model that is used to correct the trend function

The stationary Gaussian process has zero mean, constant variance and a stationary autocorrelation function $r\left(\boldsymbol{x}, \boldsymbol{x}^{\prime}\right)$. The most used class of autocorrelation functions $r\left(\boldsymbol{x}, \boldsymbol{x}^{\prime}\right)$ is the anisotropic generalized exponential model

$$
r\left(\boldsymbol{x}, \boldsymbol{x}^{\prime}\right)=\exp \left(-\sum_{k=1}^{D} \theta_{k}\left|x_{k}-x_{k}^{\prime}\right|^{\gamma}\right),
$$

where $D$ is the number of input dimensions, $\theta_{k}$ are the correlation parameters and $\gamma$ is a parameter which must satisfy $0<\gamma<2$.

\subsection{Multivariate adaptive regression splines}

The multivariate adaptive regression splines (MARS) consists of a non-parametric regression technique, first described by Friedman in 1991 [8]. This surrogate model approaches the objective function by means of a continuous surface of splines, and is of the form

$$
\hat{f}_{M A R S}(\boldsymbol{x})=\sum_{m=1}^{M_{b}} a_{m} B_{m}(\boldsymbol{x}),
$$

where $a_{m}$ are the coefficient of the power basis functions $B_{m}$ and $M_{b}$ is the number of functions.

\subsection{Artificial neural networks}

The artificial neural network (ANN) model consists of training a network of neurons by adjusting its connections weights. One of the first approaches was made by [9]. The ANN surface fitting method used in this work employs a stochastic layered perceptron (SLP) artificial neural network based on the approach of Zimmerman [10], which is of the form:

$$
\hat{f}_{A N N}(\boldsymbol{x}) \approx \tanh \left\{\tanh \left[\left(\boldsymbol{x} \boldsymbol{A}_{0}+\theta_{0}\right) \boldsymbol{A}_{1}+\theta_{1}\right]\right\}
$$

where the terms $\boldsymbol{A}_{0}, \theta_{0}, \boldsymbol{A}_{1}$ and $\theta_{1}$ are the matrices and vectors that correspond to the neuron weights and offset values in the ANN model.

\subsection{Moving least squares}

Moving least squares (MLS) is a further generation of weighted least squares where the weighting is recalculated for every new point [11]. Weighted least squares weigh the residual terms so the optimal coefficients governing the polynomial regression function $\hat{f}(\boldsymbol{x})$ are obtained by minimizing the weighted 
sum of squares at $N$ samples:

$$
\sum_{n_{1}=1}^{N} \omega_{n}\left(\left\|\hat{f}\left(\boldsymbol{x}_{\boldsymbol{n}}\right)-f\left(\boldsymbol{x}_{\boldsymbol{n}}\right)\right\|\right)
$$

\section{Application examples}

The methodologies presented are applied to two examples. The textbook test function -minimizing the $2 \mathrm{D}$ case subject to a probabilistic constraint-, and a stiffened composite panel, minimizing its weight and subject to two probabilistic constraints. These examples are carried out using PMA as RBDO methodology, and with first order reliability method (FORM) as reliability analysis method. Optimization is executed by sequential quadratic programing (SQP). The methods were implemented using DAKOTA framework [12] and the analyses were run in a high performance computing cluster with 768 computing cores, a theoretical peak performance of 5.1 TFLOP's and a physical memory of $1.8 \mathrm{~TB}$.

\subsection{Textbook function}

The problem is formulated as

$$
\min F_{T}(\boldsymbol{x})=\sum_{i=1}^{n}\left(x_{i}-1\right)^{4},
$$

subject to

$$
\begin{gathered}
G_{T}(\boldsymbol{x})=\Phi^{-1}\left(1-p\left[g_{T}(\boldsymbol{x}) \leqslant 0\right]\right)-\beta_{\text {min }} \geqslant 0, \\
-2 \leqslant x_{i} \leqslant 1,
\end{gathered}
$$

where $\beta_{\text {min }}=3$ and the limit state function $g_{T}$ is defined as

$$
g_{T}(\boldsymbol{x})=-5 \sum_{i=1}^{n} x_{i}
$$

This constraint moves the original unconstrained minimum in the chosen domain from $(1,1)$ to the deterministic optimum $(0,0)$, whereas the initial design is located at $(-1.75,-1.75)$. All the variables of the problem are considered as random design variables with normal distribution and a standard deviation of $\sigma=0.1$. The order of the surrogate models chosen for this function are of less order than the function, with the aim of avoiding the exact reproduction of the original function.

Table 1 shows the results of applying RBDO with several surrogate models. As it can be seen, all models are sensitive to the approximation order. For instance, ANN models are more accurate with an elevate number of neurons and MARS with a high number of bases, as well as MLS with a high order approximation. On the other hand, polynomials are more accurate with quadratic approximations. 
Table 1: RBDO results of the textbook function comparing accuracy and computational cost using different surrogate methods and a global sampling strategy with 20 samples.

\begin{tabular}{|c|c|c|c|c|c|c|c|c|c|}
\hline \multicolumn{2}{|c|}{ Surrogate method } & \multirow[t]{2}{*}{ Time (s) } & \multicolumn{2}{|c|}{ Evaluations } & \multirow[t]{2}{*}{ Iter. } & \multirow[t]{2}{*}{$x_{1}^{*}$} & \multirow[t]{2}{*}{$x_{2}^{*}$} & \multirow[t]{2}{*}{$F_{T}\left(\boldsymbol{x}^{*}\right)$} & \multirow[t]{2}{*}{$G_{T}\left(\boldsymbol{x}^{*}\right)$} \\
\hline Emulator & Order & & Inner & Outer & & & & & \\
\hline Non-surroga & te DO & 4.31 & - & 8 & 4 & 0.0000 & 0.0000 & 2.0000 & $-3.11 \cdot 10^{-13^{\mathrm{d}}}$ \\
\hline Non-surroga & te RBDO & 39.62 & 311 & 10 & 4 & -0.2122 & -0.2122 & 4.3179 & $2.82 \cdot 10^{-4}$ \\
\hline \multirow[t]{3}{*}{ Polynomial } & Linear & $0.15^{\mathrm{t}}$ & 354 & 14 & 5 & 1.0000 & -1.2630 & 11.6879 & $-1.62 \cdot 10^{-9}$ \\
\hline & Quadratic & $0.24^{\mathrm{t}}$ & 507 & 20 & 8 & -0.1888 & -0.2354 & 1.8130 & $-2.87 \cdot 10^{-4}$ \\
\hline & Cubic & $0.23^{\mathrm{t}}$ & 372 & 15 & 6 & -1.2630 & 1.0000 & 23.4772 & $-1.57 \cdot 10^{-9}$ \\
\hline \multirow[t]{3}{*}{ Kriging } & Constant & $0.56^{\mathrm{t}}$ & 279 & 10 & 4 & -0.2124 & -0.2119 & 4.3186 & $2.70 \cdot 10^{-4}$ \\
\hline & Linear & $21.39^{t}$ & 372 & 10 & 4 & -0.2116 & -0.2127 & 4.3182 & $2.78 \cdot 10^{-4}$ \\
\hline & Quadratic & $137.35^{\mathrm{t}}$ & 813 & 26 & 11 & -0.2125 & -0.2117 & 4.3206 & $1.82 \cdot 10^{-11}$ \\
\hline \multirow[t]{3}{*}{ MARS } & 3 bases & $0.16^{\mathrm{t}}$ & 4266 & 31 & 13 & 0.0099 & -0.4342 & 11.6510 & $-8.58 \cdot 10^{-6}$ \\
\hline & 5 bases & $0.14^{\mathrm{t}}$ & 4362 & 38 & 17 & -0.4142 & -0.0101 & 2.8604 & $5.72 \cdot 10^{-6}$ \\
\hline & 10 bases & $0.22^{\mathrm{t}}$ & 2706 & 33 & 15 & -0.3162 & -0.1081 & 4.7154 & $8.58 \cdot 10^{-6}$ \\
\hline \multirow[t]{4}{*}{ ANN } & 5 nodes & $0.24^{\mathrm{t}}$ & 1122 & 27 & 12 & -0.3320 & -0.2861 & 4.9082 & $1.89 \cdot 10^{-4}$ \\
\hline & 10 nodes & $0.12^{\mathrm{t}}$ & 2331 & 37 & 16 & -0.2032 & -0.2026 & 4.8101 & $2.00 \cdot 10^{-6}$ \\
\hline & 15 nodes & $0.26^{\mathrm{t}}$ & 1008 & 36 & 15 & -0.1986 & -0.2145 & 4.3992 & $-8.60 \cdot 10^{-6}$ \\
\hline & 18 nodes & $0.12^{\mathrm{t}}$ & 1161 & 36 & 15 & -0.2167 & -0.1955 & 4.2500 & $3.04 \cdot 10^{-6}$ \\
\hline \multirow[t]{3}{*}{ MLS } & Linear & $0.42^{\mathrm{t}}$ & 600 & 24 & 12 & -0.3925 & -0.0317 & 4.8347 & $-3.81 \cdot 10^{-10}$ \\
\hline & Quadratic & $11.30^{\mathrm{t}}$ & 789 & 28 & 13 & -0.2211 & -0.2031 & 4.4793 & $-5.70 \cdot 10^{-11}$ \\
\hline & Cubic & $4.27^{\mathrm{t}}$ & 309 & 19 & 10 & -0.2045 & -0.2199 & 4.2603 & $2.82 \cdot 10^{-4}$ \\
\hline
\end{tabular}

${ }^{\mathrm{d}}$ This value is for the constraint expressed as deterministic using $g_{T}(\boldsymbol{x}) \geqslant 0$ (eq. (10)).

${ }^{t}$ Computational times of surrogate-based RBDO do not include the sampling time, which is 4.073 seconds.

In terms of accuracy, kriging models show the best performance due to the capabilities of their correction of the trend function, which ensures that the model passes for the value of every sample. Polynomial models have the worse results, as well as MARS with a low number of bases. MARS, ANN and MLS reach good results if the order of the approximation is adequate.

In terms of computational cost, surrogate-based optimization reaches convergence in a time which is several orders of magnitude lower than the nonsurrogate case. There are some exceptions where the convergence of the surrogate model is expensive, like kriging and MLS models. However, if the simulation is expensive, the saves are notorious whatever the chosen surrogate model is.

Despite the computational improvements of this methodology, there are some points that must be had into account. It is intuitive that the surrogate model should 
give an accurate response function in the area of the domain where the RBDO process would require information. However, this is not always possible.

When random design variables are considered, accurate responses are required from both optimization algorithm and reliability analysis. Figure 1 shows the points required by both process in the whole domain. As shown, local samplings are insufficient, and global sampling requires great accuracy in several areas of the whole domain.

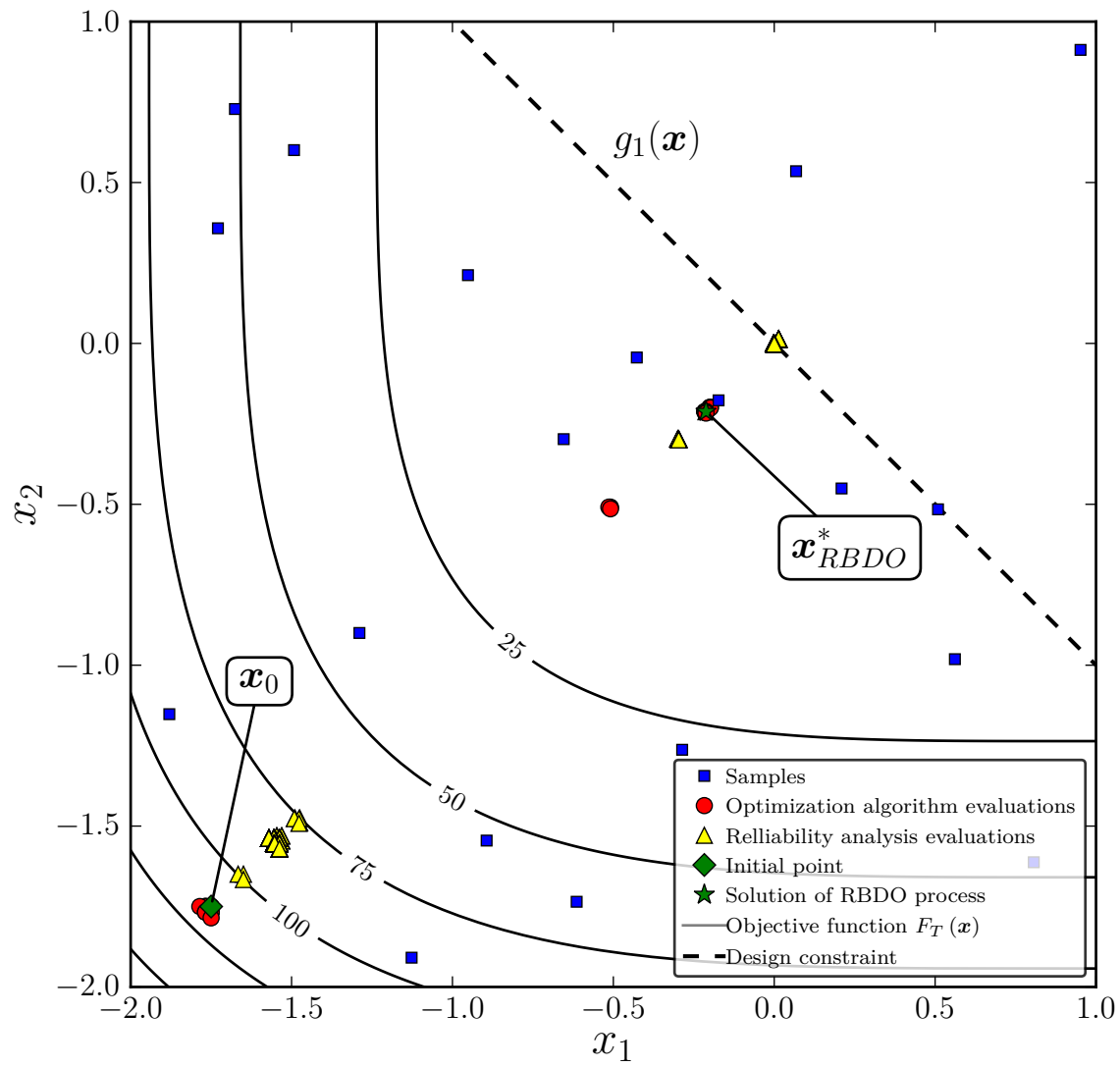

Figure 1: Textbook function in the chosen domain. The samples generated by the design of experiments process are shown, as well as the points calculated in the non-surrogate RBDO process using PMA and FORM methods.

The accuracy of the response functions is given by the design of computational experiments process and the selected surrogate model. In this case, results are 
accurate enough because the response functions are simple, but with more complex functions a high number of samples would be required to allow an accurate reliability analysis convergence.

\subsection{Stiffened composite panel}

These methods are also applied to a stiffened panel with a composite carbon/epoxy skin reinforced by four aluminum frames and stiffeners (figure 2). The structure is under an edge compression load applied along the main dimension of the panel and a shear load applied along the other two edges.

The composite material has an ultimate longitudinal strength in tension and compression of $2070 \mathrm{MPa}$ and $1160 \mathrm{MPa}$, respectively. The ultimate transverse strength in tension is $29 \mathrm{MPa}$ and $157.9 \mathrm{MPa}$ in compression. The interplane shear strength is $91 \mathrm{MPa}$. All of these quantities are considered as deterministic design parameters. The stacking sequence of the composite skin is $[ \pm 45 / 90 / 0]_{s}$ and the thickness of a stacking sequence $t$ is a random design variable with a coefficient of variation of $\delta_{t}=0.05$, an initial point of $t_{0}=5.0 \mathrm{~mm}$ and lower and upper bound of $3 \mathrm{~mm}$ and $5 \mathrm{~mm}$, respectively. Besides, the longitudinal Young's modulus $E_{1}$ is considered as random variable, with a mean value of $\mu_{E_{1}}=129.8 \mathrm{GPa}$ and a coefficient of variation of $\delta_{E_{1}}=0.05$. The frames and the stiffeners are made of aluminum with the elastic modulus of $72 \mathrm{MPa}$.

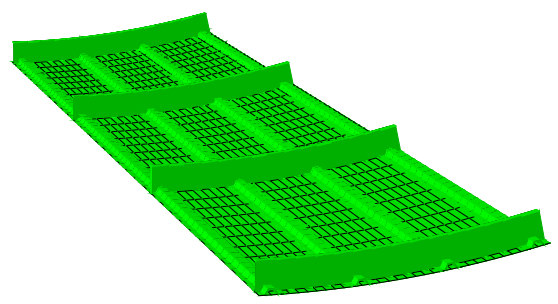

Figure 2: Finite element mesh.

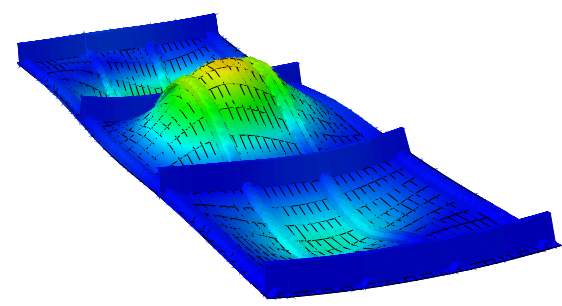

Figure 3: First buckling mode.

The objective function is the total weight of the structure and two limit state functions have been taken into account as design constraints to verify the structural reliability. The first limit state evaluates the buckling factor $\lambda$ of the first buckling mode (figure 3 ), which must be greater than a minimum value $\lambda_{\min }=100$. This condition can be expressed as

$$
g_{1}=\frac{\lambda}{\lambda_{\min }}-1 .
$$

The second limit state is based on the Tsai-Wu first ply failure criterion, which takes into consideration the interactions between different stress components and 
is expressed as

$$
\begin{aligned}
g_{2}=1-\left[\frac{\sigma_{1}^{2}}{X_{t} X_{c}}+\frac{\sigma_{2}^{2}}{Y_{t} Y_{c}}+\frac{\tau_{12}^{2}}{S^{2}}-\right. & \frac{\sigma_{1} \sigma_{2}}{\sqrt{X_{t} X_{c} Y_{t} Y_{c}}}+ \\
& \left.\left(\frac{1}{X_{t}}-\frac{1}{X_{c}}\right) \sigma_{1}+\left(\frac{1}{Y_{t}}-\frac{1}{Y_{c}}\right) \sigma_{2}\right]
\end{aligned}
$$

where $X_{c}$ and $X_{t}$ are the compressive and tensile strengths parallel to the fiber directions. $Y_{c}$ and $Y_{t}$ are the compressive and tensile strengths orthogonal to the fiber directions and $S$ is the shear strength. $\sigma_{1}, \sigma_{2}$ and $\tau_{12}$ are the stresses in material directions 1 and 2 and the shear stress, respectively. Both constraints are

Table 2: RBDO results of the stiffened composite panel comparing accuracy and computational cost using different surrogate methods and a global sampling strategy with 20 samples for the deterministic optimization and

\begin{tabular}{|c|c|c|c|c|c|c|c|c|c|}
\hline \multicolumn{2}{|c|}{ Surrogate method } & \multirow[t]{2}{*}{ Time (s) } & \multicolumn{2}{|c|}{ Evaluations } & \multirow[t]{2}{*}{ Iter. } & \multirow[t]{2}{*}{$t^{*}$} & \multirow[t]{2}{*}{$F_{T}\left(\boldsymbol{x}^{*}\right)$} & \multicolumn{2}{|c|}{ Design constraints } \\
\hline Emulator & Order & & Inner & Outer & & & & Buckling & Tsai-Wu \\
\hline Non-surrogat & te DO & 137.49 & - & 7 & 3 & 4.3975 & 7.5083 & 1.7882 & $-9.89 \cdot 10^{-5^{d}}$ \\
\hline Non-surrogat & te RBDO 56 & 318.40 & 5290 & 16 & 8 & 5.2173 & 8.7667 & 1.8804 & $-4.70 \cdot 10^{-7}$ \\
\hline \multirow[t]{3}{*}{ Polynomial } & Linear & $1.11^{\mathrm{t}}$ & 822 & 6 & 4 & 5.0000 & 8.4332 & 1.5007 & $-4.31 \cdot 10^{-2}$ \\
\hline & Quadratic & $0.65^{\mathrm{t}}$ & 6426 & 12 & 6 & 5.2147 & 8.7627 & 1.8758 & $-2.44 \cdot 10^{-4}$ \\
\hline & Cubic & $1.14^{\mathrm{t}}$ & 6738 & 12 & 6 & 5.2153 & 8.7637 & 1.8777 & $-2.39 \cdot 10^{-4}$ \\
\hline \multirow[t]{3}{*}{ Kriging } & Constant & $60.33^{\mathrm{t}}$ & 1326 & 12 & 6 & 5.2179 & 8.7676 & 1.8812 & $-2.16 \cdot 10^{-4}$ \\
\hline & Linear & $456.05^{\mathrm{t}}$ & 1170 & 12 & 6 & 5.2166 & 8.7657 & 1.8795 & $-2.99 \cdot 10^{-4}$ \\
\hline & Quadratic & $484.46^{\mathrm{t}}$ & 1437 & 12 & 6 & 5.2168 & 8.7660 & 1.8798 & $-3.00 \cdot 10^{-4}$ \\
\hline \multirow[t]{3}{*}{ MARS } & 5 bases & $0.22^{\mathrm{t}}$ & 3279 & 12 & 6 & 5.2099 & 8.7553 & 1.8440 & $-9.71 \cdot 10^{-5}$ \\
\hline & 10 bases & $0.32^{\mathrm{t}}$ & 4623 & 16 & 8 & 5.2187 & 8.7689 & 1.8879 & $5.05 \cdot 10^{-9}$ \\
\hline & 15 bases & $0.16^{\mathrm{t}}$ & 3267 & 17 & 9 & 5.2085 & 8.7533 & 1.8745 & $-8.24 \cdot 10^{-6}$ \\
\hline \multirow[t]{3}{*}{ ANN } & 10 nodes & $1.21^{\mathrm{t}}$ & 14439 & 12 & 6 & 5.2836 & 8.8703 & 1.9000 & 1.90 \\
\hline & 20 nodes & $1.18^{\mathrm{t}}$ & 2934 & 16 & 8 & 5.2218 & 8.7753 & 1.8871 & $2.79 \cdot 10^{-5}$ \\
\hline & 30 nodes & $0.66^{t}$ & 4107 & 17 & 9 & 5.2136 & 8.7612 & 2.4036 & $3.87 \cdot 10^{-4}$ \\
\hline
\end{tabular}
40 for RBDO.

\footnotetext{
${ }^{\mathrm{d}}$ This value is for the constraint expressed as deterministic using $g_{1}(\boldsymbol{x}) \geqslant 0$ (eq. (11)) and $g_{1}(\boldsymbol{x}) \geqslant 0$ (eq. (12)).

${ }^{t}$ Computational times of surrogate-based RBDO do not include the sampling time, which is 17.89 seconds for the deterministic sampling and 21.58 second in the probabilistic case.
}

defined in base of these limit state functions as

$$
G_{i}(\boldsymbol{x})=\Phi^{-1}\left(1-p\left[g_{i}(\boldsymbol{x}) \leqslant 0\right]\right)-\beta_{\min } \geqslant 0,
$$

where $\beta_{\min }=3$. 
Table 2 show the results of applying RBDO with several surrogate models in the composite panel. The inclusion of uncertainty in the stacking thickness and in the Young's modulus moves the optimum thickness from $4.39 \mathrm{~mm}$ in the deterministic case to 5.21 in the probabilistic optimization case. This time, differences between non-surrogate and surrogate approaches in terms of computational cost are very noticeable, and consequently the use of surrogate model are recommended. The increment in cost of evaluating a time-consuming simulation, makes the nonsurrogate approach less competitive. Again, kriging emulator has the slower convergence in the training process, and other models reach similar total wallclock times.

In terms of accuracy, all models reach satisfactory results if the order of the approximation is enough. For instance, a linear polynomial approximation is clearly insufficient in this example, as well as a ten nodes ANN is. But, in contrast, when more nodes are used in the ANN, and a high order polynomial is considered, accurate results are obtained. In the case of kriging model this phenomena is much less influent because these errors are avoided by the stationary Gaussian process error models $\varepsilon(\boldsymbol{x})$ (2). In contrast with the previous example, MARS model is accurate in all the approximations considered. However, a bad choice in the selection of the number of bases can lead to inaccurate results.

\section{Conclusions}

This paper presents a review of some existing procedures of surrogate modeling and their performance when are combined with reliability-based design optimization. With this aim, two examples have been employed.

As a general conclusion it can be said that the use of surrogate models represent a notable improvement in terms of computational cost. However, this improvement can be associated with a reduction of accuracy. This reduction can be prevented selecting the adequate order of the approximation and providing an enough quantity of samples of the region of interest. Specifically, this can be made by giving large data of the whole domain, or data in the neighborhoods of the solution.

In terms of accuracy, polynomial approximations give the worse results, while kriging models stand out as the more accurate. MARS, ANN and MLS, as well as polynomials, are very sensitive to the order of the approximation.

\section{Acknowledgements}

The research leading to these results has received funding from the Spanish Ministry of Science and Innovation (Ministerio de Ciencia e Innovación) under grant agreement DPI2010-16238. The authors fully acknowledge the support received. The authors also want to thank $\mathrm{PhD}$ candidate Miguel Costas Piñó for his valuable collaboration. 


\section{References}

[1] Aoues, Y. \& Chateauneuf, A., Benchmark study of numerical methods for reliability-based design optimization. Structural and Multidisciplinary Optimization, 41(2), pp. 277-294, 2009.

[2] Valdebenito, M.A. \& Schuëller, G.I., A survey on approaches for reliabilitybased optimization. Structural and Multidisciplinary Optimization, 42(5), pp. 645-663, 2010.

[3] Yao, W., Chen, X., Luo, W., Van Tooren, M. \& Guo, J., Review of uncertainty-based multidisciplinary design optimization methods for aerospace vehicles. Progress in Aerospace Sciences, 47(6), pp. 450-479, 2011.

[4] Forrester, A.I.J., Sóbester, A. \& Keane, A.J., Engineering Design via Surrogate Modelling. Wiley, 2008.

[5] Bichon, B.J., McFarland, J.M. \& Mahadevan, S., Efficient surrogate models for reliability analysis of systems with multiple failure modes. Reliability Engineering and System Safety, 96, pp. 1386-1395, 2011.

[6] Krige, D.G., A statistical approach to some basic mine valuation problems on the witwatersrand. Journal of the Chemical, Metallurgical and Mining Engineering Society of South Africa, 52(6), pp. 119-139, 1951.

[7] Sacks, J., Welch, W.J., Mitchell, T.J. \& Wynn, H., Design and analysis of computer experiments. Statistical Science, 4(4), pp. 409-423, 1989.

[8] Friedman, J.H., Multivariate adaptive regression splines. Annals of Statistics, 19, pp. 1-141, 1991.

[9] McCulloch, W. \& Pitts, W., A logical calculus of the ideas immanent in nervous activity. Bulletin of Mathematical Biophysics, 7, p. 115-133, 1943.

[10] Zimmerman, D.C., Genetic algorithms for navigating expensive and complex design spaces. Technical report, Final Report for Sandia National Laboratories contract AO-7736 CA 02, 1996.

[11] Nealen, A., A short-as-possible introduction to the least squares, weighted least squares, and moving least squares methods for scattered data approximation and interpolation. Technical report, Technical report, Discrete Geometric Modeling Group, Technishe Universitaet, Berlin, Germany, 2004.

[12] Adams, B.M., Bauman, L.E., Bohnhoff, W.J., Dalbey, K.R., Ebeida, M.S., Eddy, J.P., Eldred, M.S., Hough, P.D., Hu, K.T., Jakeman, J.D., Swiler, L.P. \& Vigil, D.M., Dakota, a multilevel parallel object-oriented framework for design optimization, parameter estimation, uncertainty quantification, and sensitivity analysis: Version 5.3.1 user's manual. Technical report, Sandia Technical Report SAND2010-2183, December 2009 Updated April 2013. 